\title{
Measurements of CP violation and the CKM matrix at $\mathrm{LHCb}$
}

\section{Marta CALVI*广}

University Milano Bicocca

E-mail: marta.calvi@mib.infn.it

Precise measurements of the CKM unitarity triangle angles are key goals of the LHCb physics programme. This paper presents the steps foreseen for the measurement of the CP-violating phase related to the $\mathrm{B}_{\mathrm{s}}^{0} \rightarrow \mathrm{J} / \psi \phi$ decay. This observable is predicted to be very small in the Standard Model, while many models of New Physics lead to significant enhancements. LHCb has the capability to improve significantly the existing experimental knowledge on this phase with the data expected in the 2010 run, and to probe down to the Standard Model prediction within a few years of operation. A short review is also given of other $\mathrm{CP}$ measurements sensitive to New Physics.

European Physical Society Europhysics Conference on High Energy Physics July 16-22, 2009

Krakow, Poland

* Speaker.

${ }^{\dagger}$ on behaf of the LHCb Collaboration. 


\section{Introduction}

All measurements of $\mathrm{CP}$ violation performed up to now in the laboratory are in good agreement with Standard Model (SM) predictions, however explanation of the observed baryon asymmetry of the universe requires larger violations. Precision measurements of the Cabibbo-Kobayshi-Maskawa (CKM) matrix elements have provided stringent test of the SM, but there is space for finding effects due to New Physics (NP) contributions especially in the $b \rightarrow s$ transitions where limited knowledge is available up to now.

B physics at LHC has the great advantage of high $b \bar{b}$ cross section: $\sigma_{b b} \sim 500 \mu b$ for $\sqrt{s}$ $=14 \mathrm{TeV}$, with production of all species of b-hadrons, while the total inelastic cross section is $\sigma_{\text {inelastic }} \sim 80 \mathrm{mb}$. About $10^{12} \mathrm{~b} \overline{\mathrm{b}}$ events will be produced in $2 \mathrm{fb}^{-1}$, which corresponds to one nominal year at the average luminosity defined for LHCb as $2 \times 10^{32} \mathrm{~cm}^{-2} \mathrm{~s}^{-1}$. Because of angular correlation in proton-proton production, about $40 \%$ of the $\mathrm{b}$ pairs will be in the forward region, in the acceptance of $\mathrm{LHCb}$, which is a single arm forward detector in the pseudo-rapidity range $1.9<\eta<4$.9. A detailed description of the detector and its performance can be found in [1].

The main focus of this talk is the measurement of $\mathrm{B}_{\mathrm{s}}^{0} \rightarrow \mathrm{J} / \psi(\mu \mu) \phi(\mathrm{KK})$ decays. A CP violating phase arises from interference between $\mathrm{B}_{\mathrm{s}}^{0}$ decay to $\mathrm{J} / \psi \phi$ either directly or via $\mathrm{B}_{\mathrm{s}}^{0}-\overline{\mathrm{B}}_{\mathrm{s}}^{0}$ oscillation. Within the SM the decay $\mathrm{B}_{\mathrm{s}}^{0} \rightarrow \mathrm{J} / \psi \phi$ is dominated by $\overline{\mathrm{b}} \rightarrow \overline{\mathrm{c}} \mathrm{s} \overline{\mathrm{s}}$ quark level transitions, hence neglecting QCD penguin contribution, the phase is: $\phi_{\mathrm{s}}^{\mathrm{SM}}=-2 \beta_{\mathrm{s}}$, where $\beta_{\mathrm{s}}=$ $\arg \left(-V_{\mathrm{ts}} V_{\mathrm{tb}}^{*} / V_{\mathrm{cs}} V_{\mathrm{cb}}^{*}\right)$ is the smallest angle of the unitarity triangle corresponding to the CKM relation: $V_{\mathrm{us}} V_{\mathrm{ub}}^{*}+V_{\mathrm{cs}} V_{\mathrm{cb}}^{*}+V_{\mathrm{ts}} V_{\mathrm{tb}}^{*}=0$. Global fits to experimental data give $2 \beta_{\mathrm{s}}=\left(-0.0360_{-0.0016}^{+0.0020}\right)$ $\operatorname{rad}[2]$. New particles could contribute to the mixing box diagram modifying the SM prediction, adding a new phase. The observable phase becomes: $\phi_{\mathrm{s}}=-2 \beta_{\mathrm{s}}+\Phi^{\mathrm{NP}}$. Recent Tevatron results give hints of deviations of $\phi_{\mathrm{s}}$ from the SM predicted value [3].

The decay $\mathrm{B}_{\mathrm{s}}^{0} \rightarrow \mathrm{J} / \psi \phi$ is a pseudo-scalar to vector-vector decay. Due to total angular momentum conservation, the final state is a superposition of three possible states with relative orbital momentum between the vector mesons $\ell=0,1,2$. An angular analysis of the decay products is required to disentangle statistically the three components of the final state. The three decay product angles $\Omega=\{\theta, \varphi, \psi\}$ can be defined as in [4] and in the transversity formalism [5] the amplitudes at $t=0, A_{0}(0)$ and $A_{\|}(0)$, are CP-even $(\ell=0,2)$, while $A_{\perp}(0)$ is CP-odd $(\ell=1)$.

The differential decay rates for $\mathrm{B}_{\mathrm{s}}^{0}$ and $\overline{\mathrm{B}}_{\mathrm{s}}^{0}$ mesons produced as flavour eigenstates at $t=0$ are given by:

$$
\frac{\mathrm{d}^{4} \Gamma\left(\mathrm{B}_{\mathrm{s}}^{0} \rightarrow \mathrm{J} / \psi \phi\right)}{\mathrm{d} t \mathrm{~d} \cos \theta \mathrm{d} \varphi \mathrm{d} \cos \psi} \equiv \frac{\mathrm{d}^{4} \Gamma}{\mathrm{d} t \mathrm{~d} \Omega} \propto \sum_{k=1}^{6} h_{k}(t) f_{k}(\Omega),
$$

the full expressions of the time dependent $h_{k}(t)$ and angular $f_{k}(\Omega)$ functions can be found in [4]. Dependence on $\phi_{\mathrm{s}}$ is present in several terms of the $h_{k}(t)$ functions, but for small $\phi_{\mathrm{s}}$ values, around the SM value, the main sensitivity comes from terms proportional to $\sin \phi_{\mathrm{s}}$. Most of these are multiplied by $\sin \left(\Delta m_{\mathrm{s}} t\right)$, hence information on $\phi_{\mathrm{s}}$ is mainly obtained from observation of the amplitude of the fast $\mathrm{B}_{\mathrm{s}}^{0}$ oscillations in the time distribution. These terms have opposite sign between $\mathrm{B}_{\mathrm{s}}^{0}$ and $\overline{\mathrm{B}}_{\mathrm{s}}^{0}$, therefore the analysis benefits significantly from flavour tagging.

\section{2. $\mathrm{B}_{\mathrm{s}}^{0} \rightarrow \mathrm{J} / \psi \phi$ measurements in LHCb}

The full LHCb Monte Carlo (MC) simulation predicts a signal yield in the $\mathrm{B}_{\mathrm{s}}^{0} \rightarrow \mathrm{J} / \psi \phi$ channel 


\begin{tabular}{|l|c|c|c|c|c|}
\hline Parameter & $\left|A_{0}(0)\right|^{2}$ & $\left|A_{\perp}(0)\right|^{2}$ & $\phi_{\mathrm{s}}(\mathrm{rad})$ & $\Gamma_{\mathrm{s}}\left(\mathrm{ps}^{-1}\right)$ & $\Delta \Gamma_{\mathrm{s}}\left(\mathrm{ps}^{-1}\right)$ \\
\hline Result & $0.599 \pm 0.002$ & $0.162 \pm 0.004$ & $-0.040 \pm 0.027$ & $0.686 \pm 0.004$ & $0.061 \pm 0.010$ \\
\hline
\end{tabular}

Table 1: The results for some fitted parameters, for a data-set equivalent to $2 \mathrm{fb}^{-1}$.
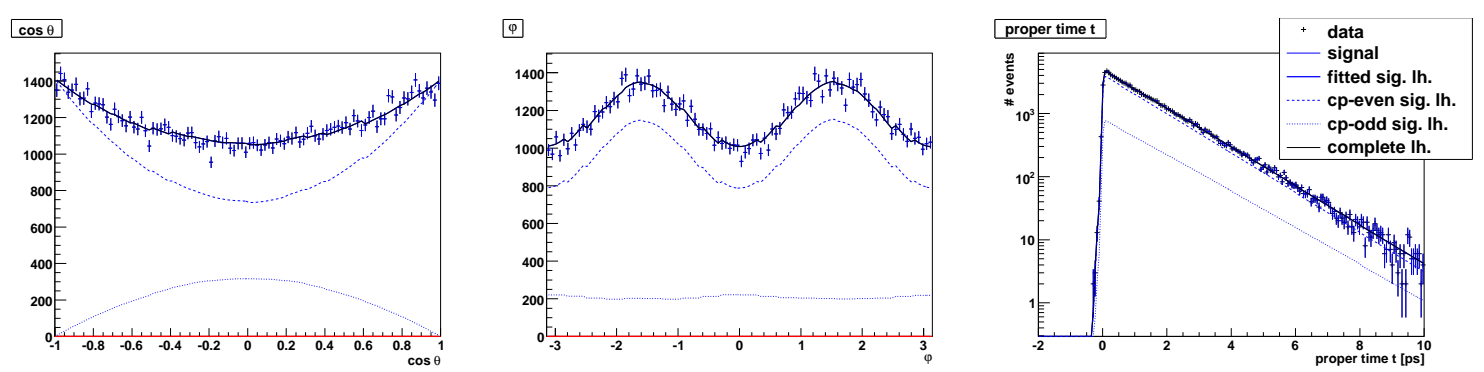

Figure 1: The projections of data and fitted signal PDF on $\cos \theta, \varphi$ and proper time, in a sample of fully simulated signal events. Also shown are the CP-even (dashed) and the CP-odd (dotted) components.

of about $120 \mathrm{k}$ events in $2 \mathrm{fb}^{-1}$, with a background over signal ratio of 0.5 for the component of long-living events. This estimation includes the trigger efficiency of about $70 \%$ for the dimuon trigger and uses an event selection designed to be lifetime-unbiased. It has the advantage of requiring small proper time and angular acceptance corrections. The background over signal ratio for the prompt $\mathrm{J} / \psi$ component is about 1.6 , but these events have negligible influence in the final event fit. Alternative analyses using lifetime-biased selections are under study and higher statistical sensitivity is anticipated.

Large use of control channels will be made at several steps in the analysis: to measure the resolution and the acceptance for the proper time and angles, and for flavour tagging calibration and measurements. Examples are $\mathrm{B}^{0} \rightarrow \mathrm{J} / \psi \mathrm{K}^{* 0}, \mathrm{~B}^{+} \rightarrow \mathrm{J} / \psi \mathrm{K}^{+}$and $\mathrm{B}_{\mathrm{s}}^{0} \rightarrow \mathrm{D}_{\mathrm{s}}^{-} \pi^{+}$decays. The expected annual yields after trigger and selection for these channels are $490 \mathrm{k}, 940 \mathrm{k}$ and $70 \mathrm{k}$ events, respectively. The flavour tagging decision will come from the combination of several methods [4]: electron, muon, kaon and inclusive secondary vertex on the "opposite side" and kaon or pion on the "same side". The total tagging power for $\mathrm{B}_{\mathrm{s}}^{0} \rightarrow \mathrm{J} / \psi \phi$ events is estimated to be $\varepsilon_{\text {tag }}(1-2 \omega)^{2}=$ $6.2 \%$, when all tagging information is combined.

The procedure for determining physics parameters from $\mathrm{B}_{\mathrm{s}}^{0} \rightarrow \mathrm{J} / \psi \phi$ data is based upon an unbinned likelihood method. Observables in the full fit are the proper time, the three decay angles, the B mass and the initial B flavour tag. Mass and proper time resolutions and angular acceptance effects are taken into account. The sensitivity to the fit parameters has been studied on full simulated MC events and with the generation of many toy MC. Good convergence on all physics parameters has been obtained and all detector parameters could also be determined from the fit. As an example, the results for some of the fitted parameters for a data-set equivalent to $2 \mathrm{fb}^{-1}$ are shown in Table 1 . The projections of data and fitted probability density function on the transversity angles and the proper time are shown in Figure 1. The fitted functions agree well with the data.

No irreducible systematic uncertainty has been identified so far. The main sources of uncertainty are possible angular distortions (relative variations of $\pm 5 \%)$, proper time resolution $\left(\sigma_{\tau}=\right.$ $(38 \pm 5) \mathrm{fs})$ and mistag rate uncertainties $(\omega=(34 \pm 1) \%)$. The resulting uncertainty $\left|\phi_{\mathrm{s}}-\phi_{\mathrm{s}}^{\text {true }}\right| / \phi_{\mathrm{s}}^{\text {true }}$ is about $7 \%$ in all three cases. A possible 5-10\% S-wave contamination in the $\phi \rightarrow \mathrm{K}^{+} \mathrm{K}^{-}$decay 

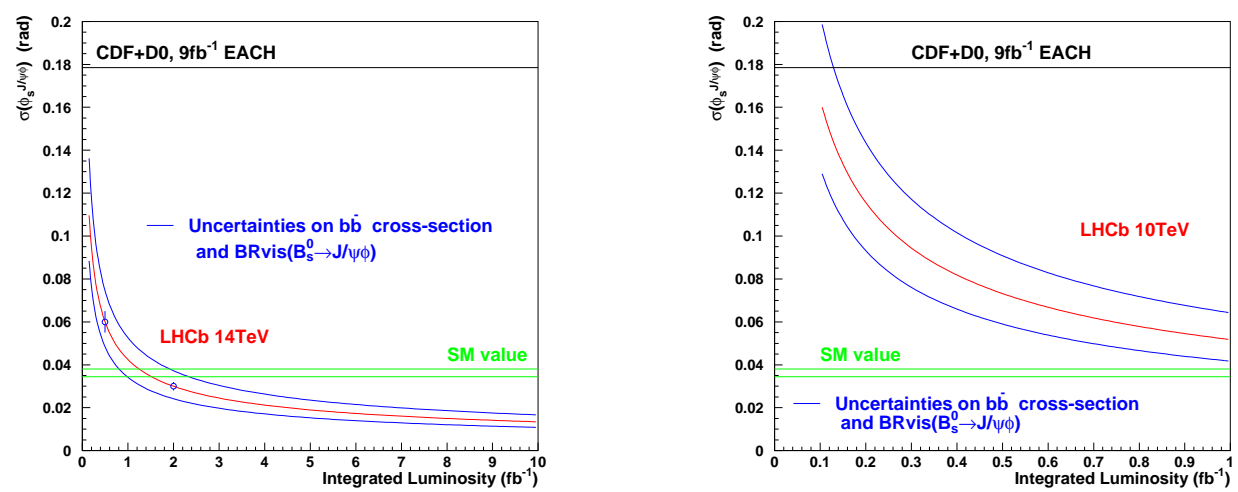

Figure 2: Statistical uncertainty on $\phi_{\mathrm{s}}$ from $\mathrm{LHCb}$ versus the integrated luminosity. The outer curves indicate the uncertainties coming from the $\mathrm{b} \overline{\mathrm{b}}$ cross-section and the visible branching ratio on $\mathrm{B}_{\mathrm{s}}^{0} \rightarrow \mathrm{J} / \psi(\mu \mu) \phi(\mathrm{KK})$. Left: from 0 to $10 \mathrm{fb}^{-1}$, assuming $\sqrt{s}=14 \mathrm{TeV}$. Right: zoom between 0 and $1 \mathrm{fb}^{-1}$, assuming $\sqrt{s}=10 \mathrm{TeV}$.

has also be considered. Ignoring this contribution in the fit will introduce a $15 \%$ bias on $\phi_{\mathrm{s}}$, while if it is included in the fit it will reduce the resolution by about $20 \%$. On the other hand, in case of a large S-wave contribution a measurement of $\cos \phi_{\mathrm{s}}$ will also become possible. Figure 2 shows the statistical uncertainty on $\phi_{\mathrm{s}}$ versus the integrated luminosity collected by LHCb. The sensitivity has been estimated for 0.5 and $2 \mathrm{fb}^{-1}$, the values are respectively $0.060 \pm 0.005$ and $0.030 \pm 0.002$. The red line is an extrapolation from these two values, assuming the errors scale like $1 / \sqrt{\mathscr{L}_{\text {int }}}$. It should be noted that for the first data, the LHC centre-of-mass energy is expected to be lower than $14 \mathrm{TeV}$, so that the $\mathrm{b} \overline{\mathrm{b}}$ cross-section will also be smaller. A centre-of-mass energy of $10 \mathrm{TeV}$ is assumed on the right plot of Figure 2.

\section{Other measurements}

Many other measurements of CKM parameters and $\mathrm{CP}$ violating variables are foreseen at LHCb. The first time dependent CP asymmetry measurement will be probably in the $\mathrm{B}^{0} \rightarrow \mathrm{J} / \psi \mathrm{K}_{\mathrm{S}}^{0}$ channel, giving a sensitivity to $\sin 2 \beta$ with the first $0.2 \mathrm{fb}^{-1}$ of about 0.06 , and of 0.02 in one nominal year. With additional data this channel will give insight into possible NP contributions to $\overline{\mathrm{b}} \rightarrow \overline{\mathrm{c}} \overline{\mathrm{s}}$ transitions and will also constrain the direct CP asymmetry. There are good perspectives at $\mathrm{LHCb}$ in the $\mathrm{B}_{\mathrm{s}}^{0} \rightarrow \phi \phi$ channel which will test possible NP contributions in $\overline{\mathrm{b}} \rightarrow \overline{\mathrm{s}} \mathrm{s} \overline{\mathrm{s}}$ penguin decays. From a time dependent analysis of the angular distribution of flavour tagged events a statistical sensitivity on the NP phase of about 0.1 is anticipated, in one nominal year. Precision results on the CKM $\gamma$ angle measurement have been presented at this Conference in [6].

\section{References}

[1] The LHCb Collaboration, The LHCb Detector at the LHC, 2008 JINST 3 S08005.

[2] J. Charles et al. (CKMfitter group), Eur. Phys. J. C41, 1-131 (2005). Updated results available at http://ckmfitter.in2p3.fr/

[3] G. Punzi, these proceedings.

[4] J. Albrecht et al. Roadmap for the measurement of mixing induced CP violation in $\mathrm{B}_{\mathrm{s}}^{0} \rightarrow \mathrm{J} / \psi \phi$ at $\mathrm{LHCb}$, LHCB-ROADMAP-002, to be published.

[5] A. Dighe et al., arXiv:hep-ph/951136v1 (1995).

[6] M. Gersabeck, these proceedings. 